UCRL-ID-121170

\title{
Parametric Fits to 1-D Neutron Transport Calculations for Lithium- Vanadium Fusion Power Plant Blankets in Cylindrical and Spherical Geometries
}

\section{Ronald W. Petzoldt and L. John Perkins \\ RECEIVED \\ OCT 161995 \\ OSTI}

June 16, 1995

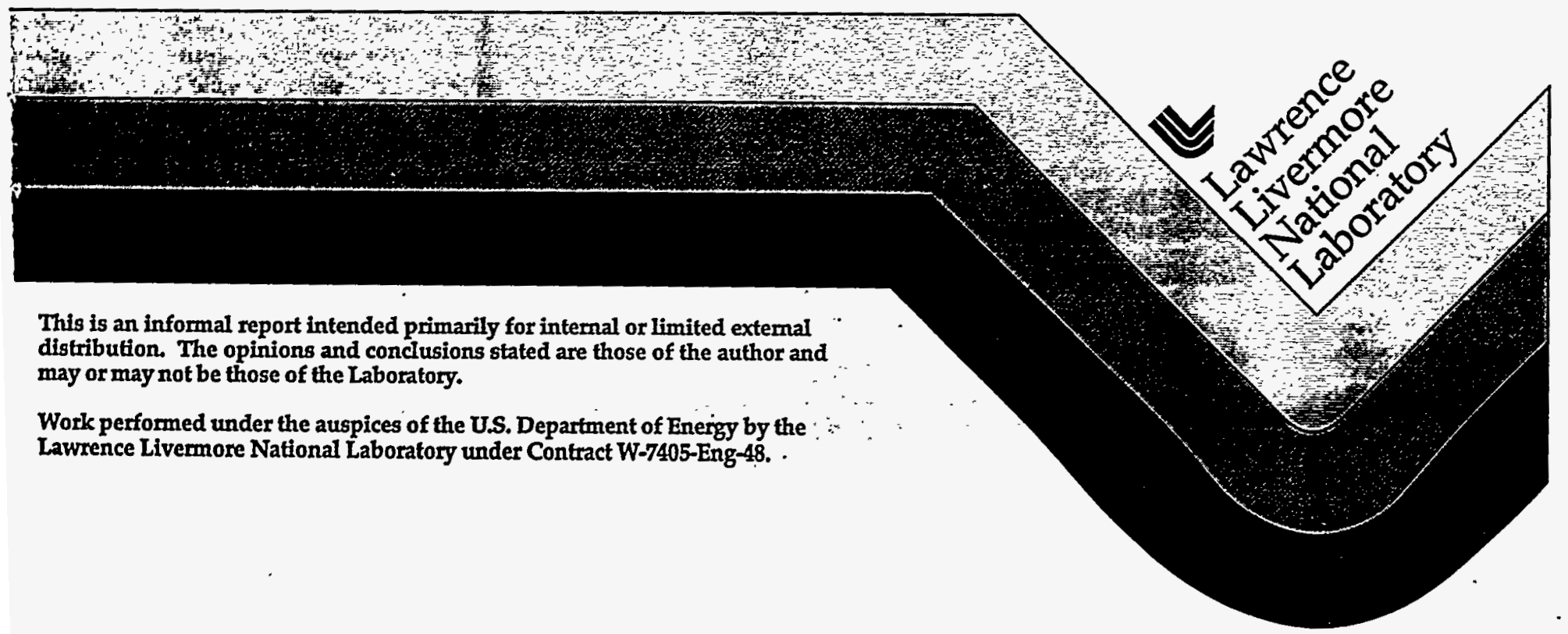

This is an informal report intended primarily for internal or limited extemal distribution. The opinions and conciusions stated are those of the author and may or may not be those of the Laboratory.

Work performed under the auspices of the U.S. Department of Energy by the Lawrence Livermore National Laboratory under Contract W-7405-Eng-48. 
This document was prepared as an account of work sponsored by an agency of the United States Government. Neither the United States Government nor the University of.California nor any of their employees, makes any warranty, express or implied, or assumes any legal liability or responsibility for the accuracy, completeness, or usefulness of any information, apparatus, product, or process disclosed, or represents that its use would not infringe privately owned rights. Reference herein to any specific commercial product, process, or service by trade name, trademark, manufacturer, or otherwise, does not necessarily constitute or imply its endorsement, recommendation, or favoring by the United States Government or the University of California. The views and opinions of authors expressed herein do not necessarily state or reflect those of the United States Government or the University of California, and shall not be used for advertising or product endorsement purposes.

This report has been reproduced directly from the best available copy.

\author{
Available to DOE and DOE contractors from the \\ Office of Scientific and Technical Information \\ P.O. Box 62, Oak Ridge, TN 37831 \\ Prices available from (615) 576-8401, FIS 626-8401
}

Available to the public from the

National Technical Information Service

U.S. Department of Commerce

5285 Port Royal Rd.,

Springfield, VA 22161

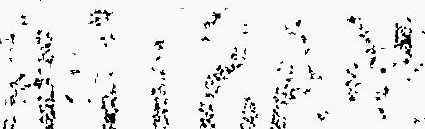




\section{DISCLAIMER}

Portions of this document may be illegible in electronic image products. Images are produced from the best available original document. 
Parametric Fits to 1-D Neutron Transport Calculations for LithiumVanadium Fusion Power Plant Blankets in Cylindrical and Spherical Geometries

Ronald W. Petzoldt and L. John.Perkins

\section{Synopsis:}

We have performed I-D coupled, neutron-gamma transport calculations for lithium-vanadium blankets and lithium-sodium cauldron "pot" blankets in cylindrical and spherical geometries. Parametric fits to the data are supplied for subsequent use in systems code models. Scaling relationships are given for various neutronics parameters of interest, including: tritium breeding ratio, neutron energy multiplication, magnet dose rates, magnet heating rates, and integrated magnet fluence.

\section{Introduction:}

We are intending to use the SuperCode ${ }^{1}$ to optimize the costs of alternate fusion power plant designs, particularly the spheromak. We wanted to calculate and optimize the size and cost of a self cooled lithium vanadium blanket with an appropriate shield for both a field reversed configuration (FRC) power plant and a spheromak power plant. We used the ONEDANT, I-D coupled neutron-gamma transport code to calculate energy deposition, neutron fluence, tritium breeding ratio, and super-conducting magnet radiation effects. ${ }^{2}$ We used a 41 neutron group, 26 gamma group library with cross-section data based on the ENDF/B-V evaluation. We used $P_{3}$ Legendre expansion order and $S_{8}$ angular quadrature. We were interested in the neutron energy multiplication factor, the total nuclear heating of cold ( $4.2 \mathrm{~K}$ ) structure and magnets, and staying below radiation and heating limits. ${ }^{3}$ The limits are:

1. $\mathrm{S} / \mathrm{C}$ magnet neutron fluence $=10^{19} \mathrm{n} / \mathrm{cm}^{2}(\mathrm{E}>0.1 \mathrm{MeV})$,

2. $\mathrm{S} / \mathrm{C}$ magnet peak volumetric heating $=2 \mathrm{~mW} / \mathrm{cm}^{3}$,

3. $\mathrm{S} / \mathrm{C}$ magnet polyamide insulation radiation $=10^{11} \mathrm{Rad}$, and

4. first wall neutron energy fluence $=16.4 \mathrm{MW}-\mathrm{yr} / \mathrm{m}^{2}$ which corresponds to $200 \mathrm{dpa}$ in the first wall structure.

We are currently using a design safety factor of 3 to account for locally higher levels of heating and fluences.

\section{System model:}

Figure 1 shows the reference case blanket, shield, and magnet model. 3 (Modifications for a cauldron blanket are discussed in a later section of this paper.) Table 1 shows the associated material compositions with atomic densities. Table 2 shows the volume percents of materials within each zone. 3 


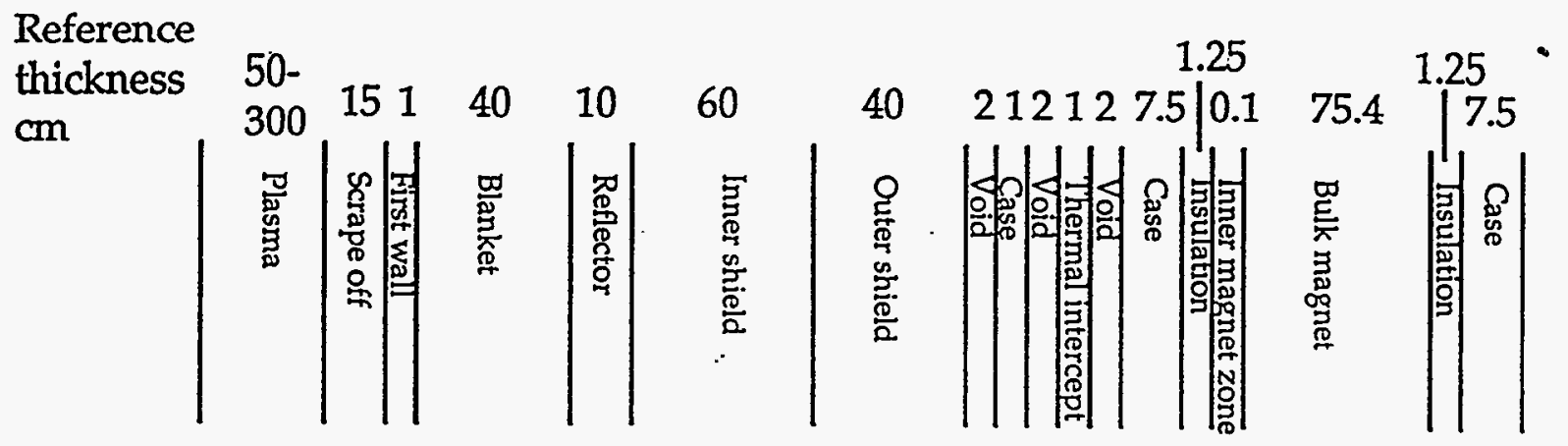

Figure 1. Reference case blanket, shield, and magnet model. 
Table 1. Material compositions.

\begin{tabular}{|c|c|c|}
\hline Material Name & Component & $\begin{array}{l}\text { Atom density } \\
10^{22} / \mathrm{cm}^{3}\end{array}$ \\
\hline \multirow[t]{9}{*}{ Incoloy } & $\overline{\mathrm{Fe}}$ & 3.556 \\
\hline & $\mathrm{Ni}$ & 4.078 \\
\hline & $\overline{\mathrm{Cr}}$ & .352 \\
\hline & $\overline{\mathrm{Nb}}$ & .155 \\
\hline & $\overline{\mathrm{Ti}}$ & .107 \\
\hline & $\overline{\mathrm{Al}}$ & .186 \\
\hline & $\overline{\mathrm{Si}}$ & .024 \\
\hline & $\overline{\mathrm{Mn}}$ & .004 \\
\hline & $\bar{C}$ & .004 \\
\hline Liquid helium & $\overline{\mathrm{He}}$ & 2.108 \\
\hline \multirow[t]{2}{*}{$\mathrm{Nb}_{3} \mathrm{Sn}$} & $\overline{\mathrm{Nb}}$ & 4.046 \\
\hline & $\overline{S n}$ & 1.3486 \\
\hline Tin & Sn & 2.92 \\
\hline Vanadium & $\bar{V}$ & 7.046 \\
\hline Copper & $\overline{\mathrm{Cu}}$ & 8.434 \\
\hline Aluminum & $\overline{\mathrm{Al}}$ & 6.03 \\
\hline \multirow[t]{7}{*}{ Polyamide } & $\mathrm{Si}$ & 0.8765 \\
\hline & $0-16$ & 2.898 \\
\hline & $\mathrm{Al}$ & 0.3966 \\
\hline & $\overline{\mathrm{Mg}}$ & 0.2 \\
\hline & $\mathrm{C}$ & 2.12 \\
\hline & $\mathrm{H}-1$ & 1.746 \\
\hline & $\mathrm{N}$ & 0.35 \\
\hline Liquid nitrogen & $\mathrm{N}$ & 3.474 \\
\hline \multirow[t]{5}{*}{ Tenelon } & $\overline{\mathrm{Fe}}$ & 6.0 \\
\hline & $\mathrm{Mn}$ & 1.314 \\
\hline & $\mathrm{Cr}$ & 1.39 \\
\hline & $\mathrm{Si}$ & 0.0343 \\
\hline & $\bar{C}$ & 0.06 \\
\hline \multirow[t]{2}{*}{ Lithium } & $\mathrm{Li}-6$ & 0.316 \\
\hline & Li-7 & 3.954 \\
\hline \multirow[t]{3}{*}{ Vanadium alloy } & $\mathrm{V}$ & 6.384 \\
\hline & $\overline{\mathrm{Cr}}$ & 0.347 \\
\hline & $\overline{\mathrm{Ti}}$ & 0.377 \\
\hline \multirow[t]{7}{*}{ Borated tenelon } & $\mathrm{Fe}$ & 5.57 \\
\hline & $\overline{\mathrm{Mn}}$ & 1.314 \\
\hline & $\overline{\mathrm{Cr}}$ & 1.39 \\
\hline & $\overline{\mathrm{Si}}$ & 0.0343 \\
\hline & $\bar{C}$ & 0.06 \\
\hline & B-10 & 0.257 \\
\hline & B-11 & 1.04 \\
\hline
\end{tabular}


Table 2. Zone compositions

\begin{tabular}{|l|l|l|}
\hline Model zone & Material & Volume percent \\
\hline Magnet case & Incoloy & 97 \\
\hline & Liquid helium & 3 \\
\hline Insulation & Polyamide & 100 \\
\hline Magnet & Incoloy & 25 \\
\hline & Copper & 27.32 \\
\hline & Nb3Sn & 3.42 \\
\hline & Tin & 2.37 \\
\hline & Vanadium & 1.89 \\
\hline & Liquid helium & 15 \\
\hline & Polyamide & 25 \\
\hline Thermal intercept & Aluminum & 70 \\
\hline & Liquid nitrogen & 20 \\
\hline First wall & Lithium & 71.4 \\
\hline & Vanadium alloy & 28.6 \\
\hline Blanket & Lithium & 90 \\
\hline & Vanadium alloy & 10 \\
\hline Reflector & Vanadium alloy & 15 \\
\hline & Lithium & 10 \\
\hline & Tenelon & 75 \\
\hline Inner shield & Vanadium alloy & 15 \\
\hline & Lithium & 5 \\
\hline & Tenelon & 80 \\
\hline Outer shield & Tenelon & 15 \\
\hline & Borated tenelon & 80 \\
\hline & Lithium & 5 \\
\hline
\end{tabular}

Method and results:

1-D coupled neutron-gamma transport calculations were performed to determine the effects of varying first wall radius, and blanket, inner shield, and outer shield thicknesses for cylindrical and spherical geometries applicable to the FRC and spheromak respectively. Calculations were normalized to $1 \mathrm{MW} / \mathrm{m}^{2}$ first wall neutron load, and 30 full power years of operation. Appendix 1 shows typical data collected on each run. Tables 3 and 4 show selected data for cylindrical and spherical geometries respectively. We did inner shield variations for the cylindrical symmetry only. Only one zone width is modified at a time with the rest held to the reference width's shown in Figure 1. The reference cylindrical first wall radius is $115 \mathrm{~cm}$ and the reference spherical first wall radius is $315 \mathrm{~cm}$.

Tables 3 and 4 use the following terminology. "Width" is the width of the zone being varied, " $4.2 \mathrm{~K}$ heat" is the nuclear heating of the magnet and structure that must be maintained at $4.2 \mathrm{~K}$, "Neutron fluence" is the inner magnet zone neutron fluence $(E>0.1 \mathrm{MeV})$, "Rad dose" is the inner magnet zone dose, "Local $4.2 \mathrm{~K}$ " is the inner magnet zone heating rate per unit 
volume, "TBR" is the tritium breeding ratio, and "M" is the neutron energy multiplication factor.

Table 3. Cylindrical geometry selected variational data.

Neutron wall load $=1 \mathrm{MW} / \mathrm{m}^{2}$. Lifetime $=30$ full power years.

\begin{tabular}{|l|l|l|l|l|l|l|}
\hline $\begin{array}{l}\text { Width } \\
(\mathrm{cm})\end{array}$ & $\begin{array}{l}4.2 \mathrm{~K} \text { heat } \\
(\mathrm{W} / \mathrm{cm})\end{array}$ & $\begin{array}{l}\text { Neutron } \\
\text { fluence } \\
\left(\mathrm{E}_{\mathrm{n}}>0.1\right. \\
\mathrm{MeV}) \\
\mathrm{n} / \mathrm{cm}^{2}\end{array}$ & $\begin{array}{l}\text { Rad dose } \\
(\mathrm{Rad})\end{array}$ & $\begin{array}{l}\text { Local } \\
4.2 \mathrm{~K} \\
\left(\mathrm{~W} / \mathrm{cm}^{3}\right)\end{array}$ & TBR & $\mathrm{M}$ \\
\hline Blanket & & & & & & \\
\hline 25 & 0.57 & $1.68 \times 10^{17}$ & $2.74 \times 10^{8}$ & $1.70 \times 10^{-5}$ & 0.99 & 1.45 \\
\hline 40 & 0.32 & $8.9 \times 10^{16}$ & $1.46 \times 10^{8}$ & $9.08 \times 10^{-6}$ & 1.21 & 1.38 \\
\hline 55 & 0.18 & $4.64 \times 10^{16}$ & $7.69 \times 10^{7}$ & $4.80 \times 10^{-6}$ & 1.36 & 1.32 \\
\hline $\begin{array}{l}\text { Inner } \\
\text { shield }\end{array}$ & & & & & & \\
\hline 40 & 2.96 & $1.03 \times 10^{18}$ & $1.50 \times 10^{9}$ & $9.06 \times 10^{-5}$ & 1.21 & 1.38 \\
\hline 50 & 0.982 & $3.05 \times 10^{17}$ & $4.75 \times 10^{8}$ & $2.91 \times 10^{-5}$ & 1.21 & 1.38 \\
\hline 60 & 0.32 & $8.9 \times 10^{16}$ & $1.46 \times 10^{8}$ & $9.08 \times 10^{-6}$ & 1.21 & 1.38 \\
\hline $\begin{array}{l}\text { Outer } \\
\text { shield }\end{array}$ & & & & & & \\
\hline 20 & 4.81 & $1.59 \times 10^{18}$ & $2.41 \times 10^{9}$ & $1.47 \times 10^{-4}$ & 1.21 & 1.38 \\
\hline 30 & 1.26 & $3.81 \times 10^{17}$ & $6.00 \times 10^{8}$ & $3.72 \times 10^{-5}$ & 1.21 & 1.38 \\
\hline 40 & 0.32 & $8.90 \times 10^{16}$ & $1.46 \times 10^{8}$ & $9.08 \times 10^{-6}$ & 1.21 & 1.38 \\
\hline 50 & 0.078 & $2.02 \times 10^{16}$ & $3.46 \times 10^{7}$ & $2.15 \times 10^{-6}$ & 1.21 & 1.38 \\
\hline First & & & & & & \\
\hline wall & $\begin{array}{l}5.04 \\
65\end{array}$ & $6.89 \times 10^{16}$ & $1.14 \times 10^{8}$ & $7.07 \times 10^{-6}$ & 1.19 & 1.39 \\
\hline 115 & 4.45 & $8.9 \times 10^{16}$ & $1.46 \times 10^{8}$ & $9.08 \times 10^{-6}$ & 1.21 & 1.38 \\
\hline
\end{tabular}


Table 4. Spherical geometry selected variational data.

Neutron wall load $=1 \mathrm{MW} / \mathrm{m}^{2}$. Lifetime $=30 \mathrm{full}$ power years.

\begin{tabular}{|l|l|l|l|l|l|l|}
\hline $\begin{array}{l}\text { Width } \\
(\mathrm{cm})\end{array}$ & $\begin{array}{l}4.2 \mathrm{~K} \text { heat } \\
(\mathrm{W})\end{array}$ & $\begin{array}{l}\text { Neutron } \\
\text { fluence } \\
\left(\mathrm{E}_{\mathrm{n}}>0.1\right. \\
\mathrm{MeV}) \\
\mathrm{n} / \mathrm{cm}^{2}\end{array}$ & $\begin{array}{l}\text { Rad dose } \\
(\mathrm{Rad})\end{array}$ & $\begin{array}{l}\text { Local } \\
4.2 \mathrm{~K} \\
\left(\mathrm{~W} / \mathrm{cm}^{3}\right)\end{array}$ & TBR & $\mathrm{M}$ \\
\hline Blanket & & & & & & \\
\hline 25 & 1148 & $2.11 \times 10^{17}$ & $3.36 \times 10^{8}$ & $2.09 \times 10^{-5}$ & 0.95 & 1.46 \\
\hline 40 & 660 & $1.12 \times 10^{17}$ & $1.82 \times 10^{8}$ & $1.13 \times 10^{-5}$ & 1.18 & 1.40 \\
\hline 55 & 375 & $5.91 \times 10^{16}$ & $9.66 \times 10^{7}$ & $6.02 \times 10^{-6}$ & 1.34 & 1.33 \\
\hline $\begin{array}{l}\text { Outer } \\
\text { shield }\end{array}$ & & & & & & \\
\hline 20 & 9792 & $2.01 \times 10^{18}$ & $2.99 \times 10^{9}$ & $1.82 \times 10^{-4}$ & 1.18 & 1.40 \\
\hline 30 & 2585 & $4.81 \times 10^{17}$ & $7.47 \times 10^{8}$ & $4.62 \times 10^{-5}$ & 1.18 & 1.40 \\
\hline 40 & 660 & $1.12 \times 10^{17}$ & $1.82 \times 10^{8}$ & $1.13 \times 10^{-5}$ & 1.18 & 1.40 \\
\hline $\begin{array}{l}\text { First } \\
\text { Wall }\end{array}$ & & & & & & \\
\hline 165 & $\begin{array}{l}6.49 \\
\mathrm{~W} / \mathrm{MW}\end{array}$ & $7.92 \times 10^{16}$ & $1.29 \times 10^{8}$ & $8.01 \times 10^{-6}$ & 1.15 & 1.41 \\
\hline 315 & $\begin{array}{l}5.3 \\
\mathrm{~W} / \mathrm{MW}\end{array}$ & $1.12 \times 10^{17}$ & $1.82 \times 10^{8}$ & $1.13 \times 10^{-5}$ & 1.18 & 1.40 \\
\hline
\end{tabular}

We can make some rather expected general statements regarding the data in Tables 3 and 4. Increasing blanket thickness increases the tritium breeding ratio and decreases the energy multiplication factor. The decrease in energy multiplication factor is probably due to an endothermic $n-2 n$ reaction with ${ }^{7} \mathrm{Li}$. Increasing the thickness of any shielding material, reduces magnet radiation and heating. Changing the blanket thickness has less effect on shielding than changing the inner shield thickness which has less effect than changing the outer shield thickness. An increased first wall radius significantly reduces the effectiveness of a given thickness of shielding due to $1 / r$ or $1 / r^{2}$ geometrical effects. However, for a given shielding thickness, the shielding mass per unit first wall area is much larger for smaller first wall radii.

Tables 5 and 6 show the results of least squares fits of the data in Tables 3 and 4 . We used exponential fits where appropriate, and linear fits elsewhere. These fits are approximately correct over the range of data from which they were taken for reference values of data except for the one that is being varied. They are also expected to give approximate values when used to calculate the effect of changing several thicknesses at once. 
Table 5. Cylindrical Geometry: Least squares fits for varying zone thicknesses. Neutron wall load $=1 \mathrm{MW} / \mathrm{m}^{2}$. Lifetime $=30$ full power years.

\begin{tabular}{|l|l|}
\hline Blanket thickness $x_{b}(\mathrm{~cm})$ & \\
\hline Energy multiplication factor & $1.55-0.0043 x_{\mathrm{b}}$ \\
\hline Tritium breeding ratio & $0.693+0.0123 \mathrm{x}_{\mathrm{b}}$ \\
\hline $4.2 \mathrm{~K}$ heat $(\mathrm{W} / \mathrm{cm})$ & $1.48 \exp \left(-0.0384 \mathrm{x}_{\mathrm{b}}\right)$ \\
\hline Magnet neutron fluence $\left(\mathrm{n} / \mathrm{cm}^{2}\right)$ & $4.92 \times 10^{17} \exp \left(-0.0429 \mathrm{x}_{\mathrm{b}}\right)$ \\
\hline Magnet radiation dose $(\mathrm{Rad})$ & $7.91 \times 10^{8} \exp \left(-0.04235 \mathrm{x}_{\mathrm{b}}\right)$ \\
\hline Inner magnet zone heating $\left(\mathrm{W} / \mathrm{cm}^{3}\right)$ & $4.88 \times 10^{-5} \exp \left(-0.04215 \mathrm{x}_{\mathrm{b}}\right)$ \\
\hline & \\
\hline Inner shield thickness $\mathrm{x}_{\mathrm{i}}(\mathrm{cm})$ & \\
\hline $4.2 \mathrm{~K}$ heat $(\mathrm{W} / \mathrm{cm})$ & $254 \exp \left(-0.11123 \mathrm{x}_{\mathrm{i}}\right)$ \\
\hline Magnet neutron fluence $\left(\mathrm{n} / \mathrm{cm}^{2}\right)$ & $1.38 \times 10^{20} \exp \left(-0.11648 \mathrm{x}_{\mathrm{i}}\right)$ \\
\hline Magnet radiation dose $(\mathrm{Rad})$ & $1.59 \times 10^{11} \exp \left(-0.11502 \mathrm{x}_{\mathrm{i}}\right)$ \\
\hline Inner magnet zone heating $\left(\mathrm{W} / \mathrm{cm}^{3}\right)$ & $9.06 \times 10^{-3} \exp \left(-0.11502 \mathrm{x}_{\mathrm{i}}\right)$ \\
\hline & \\
\hline Outer shield thickness $\mathrm{x}_{\mathrm{o}}(\mathrm{cm})$ & \\
\hline $4.2 \mathrm{~K}$ heat $(\mathrm{W} / \mathrm{cm})$ & $76.35 \exp \left(-0.1374 \mathrm{x}_{\mathrm{o}}\right)$ \\
\hline Magnet neutron fluence $\left(\mathrm{n} / \mathrm{cm}^{2}\right)$ & $2.96 \times 10^{19} \exp \left(-0.1455 \mathrm{x}_{\mathrm{o}}\right)$ \\
\hline Magnet radiation dose $(\mathrm{Rad})$ & $4.13 \times 10^{10} \exp \left(-0.1414 \mathrm{x}_{0}\right)$ \\
\hline Inner magnet zone heating $\left(\mathrm{W} / \mathrm{cm}^{3}\right)$ & $2.50 \times 10^{-3} \exp \left(-0.1408 \mathrm{x}_{\mathrm{o}}\right)$ \\
\hline & \\
\hline First wall radius $\mathrm{r}_{\mathrm{W}}(\mathrm{cm})$ & $5.81-0.0118 \mathrm{r}_{\mathrm{W}}$ \\
\hline $4.2 \mathrm{~K}$ heat $(\mathrm{W} / \mathrm{MW})$ & $\left(4.28+0.0402 \mathrm{r}_{\mathrm{W}}\right) \times 10^{16}$ \\
\hline Magnet neutron fluence $\left(\mathrm{n} / \mathrm{cm}^{2}\right)$ & $\left(7.24+0.064 \mathrm{r}_{\mathrm{W}}\right) \times 10^{7}$ \\
\hline Magnet radiation dose $(\mathrm{Rad})$ & $(4.46+0.0402) \times 10^{-6}$ \\
\hline Inner magnet zone heating $\left(\mathrm{W} / \mathrm{cm}^{3}\right)$ & \\
\hline
\end{tabular}


Table 6. Spherical Geometry: Least squares fits for varying zone thicknesses. Neutron wall load $=1 \mathrm{MW} / \mathrm{m}^{2}$. Lifetime $=30$ full power years.

Blanket thickness $\mathrm{x}_{\mathrm{b}}(\mathrm{cm})$

Energy multiplication factor

Tritium breeding ratio

$4.2 \mathrm{~K}$ heat $(W)$

Magnet neutron fluence $\left(\mathrm{n} / \mathrm{cm}^{2}\right)$

Magnet radiation dose ( $\mathrm{Rad})$

Inner magnet zone heating $\left(W / \mathrm{cm}^{3}\right)$

$1.57-0.00433 x_{b}$

$0.637+0.013 x_{b}$

$2922 \exp \left(-0.0373 x_{\mathrm{b}}\right)$

$6.09 \times 10^{17} \exp \left(-0.04242 x_{b}\right)$

$9.53 \times 10^{8} \exp \left(-0.04155 x_{b}\right)$

$5.91 \times 10^{-5} \exp \left(-0.04149 x_{b}\right)$

Outer shield thickness $x_{0}(\mathrm{~cm})$

$4.2 \mathrm{~K}$ heat $(\mathrm{W})$

$1.46 \times 10^{5} \exp \left(-0.1348 \mathrm{x}_{0}\right)$

Magnet neutron fluence $\left(\mathrm{n} / \mathrm{cm}^{2}\right)$

$3.62 \times 10^{19} \exp \left(-0.1444 x_{0}\right)$

Magnet radiation dose (Rad)

$4.93 \times 10^{10} \exp \left(-0.1400 x_{0}\right)$

\begin{tabular}{ll} 
Inner magnet zone heating $\left(\mathrm{W} / \mathrm{cm}^{3}\right)$ & $2.95 \times 10^{-3} \exp \left(-0.1390 \mathrm{x}_{0}\right)$ \\
\hline
\end{tabular}

First wall radius $\mathrm{r}_{\mathrm{w}}(\mathrm{cm})$

\begin{tabular}{|l|l|}
\hline $4.2 \mathrm{~K}$ heat $(\mathrm{W} / \mathrm{MW})$ & $7.8-0.00793 \mathrm{r}_{\mathrm{W}}$ \\
\hline Magnet neutron fluence $\left(\mathrm{n} / \mathrm{cm}^{2}\right)$ & $\left(4.31+0.02187 \mathrm{r}_{\mathrm{W}}\right) \times 10^{16}$ \\
\hline Magnet radiation dose $(\mathrm{Rad})$ & $\left(7.07+0.03533 \mathrm{r}_{\mathrm{W}}\right) \times 10^{7}$ \\
\hline Inner magnet zone heating $\left(\mathrm{W} / \mathrm{cm}^{3}\right)$ & $\left(4.39+0.02193 \mathrm{r}_{\mathrm{W}}\right) \times 10^{-6}$ \\
\hline
\end{tabular}

Use of dpa data:

The first wall fluence limit of $16.4 \mathrm{MW}$ years corresponds to approximately 200 dpa in the vanadium structure. ${ }^{3}$ We obtained information on the displacements per atom (dpa) of copper that would occur for a given neutron fluence based on displacement cross sections for copper. We did not have this information for vanadium. Copper dpa is reduced roughly by a factor of 2 by $20 \mathrm{~cm}$ of blanket material. Although the actual cross-sections for displacements in vanadium will vary from those in copper, we extrapolate the results for and expect that blanket vanadium material $20 \mathrm{~cm}$ into the blanket would experience about half the number of dpa's, last about twice as long as first wall material, and only have to be replaced about half as often. Calculations using proper vanadium dpa cross-sections should be done verify this conclusion. 


\section{Example:}

Suppose we desired to calculate the inner magnet zone neutron fluence $(\phi)$ that would occur in a power plant with the following parameters.

Geometry

First wall radius

Neutron wall load $\left(P_{n}\right)$

Full power operation

Blanket thickness

Inner shield thickness

Outer shield thickness
Cylindrical

$95 \mathrm{~cm}$

$4 \mathrm{MW} / \mathrm{m}^{2}$

30 years

$35 \mathrm{~cm}$

$50 \mathrm{~cm}$

$30 \mathrm{~cm}$

We will use the base equation from table 5 for the first wall radius, modified with differences from the reference case for the neutron wall load, and blanket, inner shield, and outer shield thicknesses.

$$
\begin{aligned}
\phi= & \frac{P_{n}}{P_{n o}}\left(4.28+\lambda_{w} r_{w}\right) \times 10^{16} \mathrm{n} / \mathrm{cm}^{2} \exp \left(-\lambda_{b} \Delta x_{b}\right) \exp \left(-\lambda_{i} \Delta x_{i}\right) \exp \left(-\lambda_{o} \Delta x_{o}\right) \\
= & \frac{4}{1}(4.28+0.0402 \times 95) \times 10^{16} \mathrm{n} / \mathrm{cm}^{2} \exp (-0.0429 \times(35-40)) \\
& \exp (-0.11648 \times(50-60)) \exp (-0.1455 \times(30-40)) \\
= & 4 \times 8.099 \times 10^{16} \mathrm{n} / \mathrm{cm}^{2} \times 1.24 \times 3.21 \times 4.28 \\
= & 5.52 \times 10^{18} \mathrm{n} / \mathrm{cm}^{2} .
\end{aligned}
$$

How much thicker would the outer shield have to be to allow a design safety factor of 3 below the fluence limit of $10^{19} \mathrm{n} / \mathrm{cm}^{2}$ ?

$$
\begin{aligned}
& \phi=\phi_{0} \exp (-\lambda \Delta x) \Rightarrow \\
& \Delta x=\frac{\ln \left(\phi_{0} / \phi\right)}{\lambda}=\frac{\ln (5.52 / 3.33)}{0.1455 / \mathrm{cm}}=3.47 \mathrm{~cm}
\end{aligned}
$$

for a total outer shield thickness of $33.5 \mathrm{~cm}$.

\section{Cauldron blanket:}

A cauldron blanket similar to reference 4 was also considered for use as a spheromak blanket. The blanket consists of 50 weight percent each of molten sodium and lithium. We assume a $3 \mathrm{~m}$ radius plasma with a $15 \mathrm{~cm}$ scrape-off layer. The reference case first wall, blanket, inner shield, and outer shield thicknesses are changed to $4 \mathrm{~cm}, 150 \mathrm{~cm}, 30 \mathrm{~cm}$, and $20 \mathrm{~cm}$ respectively. The other material thickness are as shown in Figure 1. The lithium in the first wall, reflector, and shield are replaced by equal volume percents of sodium.

We used ONEDANT to perform neutronics calculations for the assumed spherical symmetry. Again we calculated the effects of varying blanket and 
inner shield thicknesses for spherical geometry. Calculations were normalized to $1 \mathrm{MW} / \mathrm{m}^{2}$ first wall neutron load, and $30 \mathrm{MW}$ years of operation. Table 7 shows selected data from the ONEDANT calculations. Only one zone width is modified at a time with the rest held to the reference width's. Tables 8 shows the results of least squares fits of the data in table 7 .

We also did a comparison calculation with a 67 weight percent sodium 33 weight percent lithium blanket. The shielding characteristics were nearly the same, but the tritium breeding ratio dropped from 1.36 to 1.18 . This may be a more desirable blanket mixture since it is the sodium that we are vaporizing for heat transfer purposes.

Table 7. Spherical Geometry:

Cauldron blanket selected variational data.

\begin{tabular}{|l|l|l|l|l|l|l|}
\hline $\begin{array}{l}\text { Width } \\
(\mathrm{cm})\end{array}$ & $\begin{array}{l}4.2 \mathrm{~K} \text { heat } \\
\text { (W) }\end{array}$ & $\begin{array}{l}\text { Neutron } \\
\text { fluence } \\
\mathrm{n} / \mathrm{cm}^{2}\end{array}$ & $\begin{array}{l}\text { Rad dose } \\
(\mathrm{Rad})\end{array}$ & $\begin{array}{l}\text { Local } \\
4.2 \mathrm{~K} \\
\left(\mathrm{~W} / \mathrm{cm}^{3}\right)\end{array}$ & $\mathrm{TBR}$ & $\mathrm{M}$ \\
\hline Blanket & & & & & & \\
\hline 130 & 17635 & $3.48 \times 10^{18}$ & $4.39 \times 10^{9}$ & $2.54 \times 10^{-4}$ & 1.33 & 1.21 \\
\hline 150 & 9229 & $1.99 \times 10^{18}$ & $2.12 \times 10^{9}$ & $1.23 \times 10^{-4}$ & 1.36 & 1.20 \\
\hline $\begin{array}{l}\text { Inner } \\
\text { shield }\end{array}$ & & & & & & \\
\hline 30 & 9229 & $1.99 \times 10^{18}$ & $2.12 \times 10^{9}$ & $1.23 \times 10^{-4}$ & 1.36 & 1.20 \\
\hline 50 & 1242 & $1.72 \times 10^{17}$ & $2.54 \times 10^{8}$ & $1.55 \times 10^{-5}$ & 1.36 & 1.20 \\
\hline
\end{tabular}

Table 8. Spherical Geometry:

Least squares fits for varying cauldron zone thicknesses.

\begin{tabular}{|l|l|}
\hline Blanket thickness $\mathbf{x}_{\mathbf{b}}(\mathrm{cm})$ & \\
\hline $4.2 \mathrm{~K}$ heat $(\mathrm{W})$ & $1.19 \times 10^{6} \exp \left(-0.03238 \mathrm{x}_{\mathrm{b}}\right)$ \\
\hline Magnet neutron fluence $\left(\mathrm{n} / \mathrm{cm}^{2}\right)$ & $1.32 \times 10^{20} \exp \left(-0.02794 \mathrm{x}_{\mathrm{b}}\right)$ \\
\hline Magnet radiation dose $(\mathrm{Rad})$ & $4.98 \times 10^{11} \exp \left(-0.03634 \mathrm{x}_{\mathrm{b}}\right)$ \\
\hline Inner magnet heating $\left(\mathrm{W} / \mathrm{cm}^{3}\right)$ & $2.83 \times 10^{-2} \exp \left(-0.03626 \mathrm{x}_{\mathrm{b}}\right)$ \\
\hline & \\
\hline Inner Shield thickness $\mathrm{x}_{\mathbf{i}}(\mathrm{cm})$ & \\
\hline $4.2 \mathrm{~K}$ heat $(\mathrm{W})$ & $1.87 \times 10^{5} \exp \left(-0.1003 \mathrm{x}_{\mathrm{i}}\right)$ \\
\hline Magnet neutron fluence $\left(\mathrm{n} / \mathrm{cm}^{2}\right)$ & $7.83 \times 10^{19} \exp \left(-0.1224 \mathrm{x}_{\mathbf{i}}\right)$ \\
\hline Magnet radiation dose $(\mathrm{Rad})$ & $5.11 \times 10^{10} \exp \left(-0.1061 \mathrm{x}_{\mathrm{i}}\right)$ \\
\hline Inner magnet heating $\left(\mathrm{W} / \mathrm{cm}^{3}\right)$ & $2.75 \times 10^{-3} \exp \left(-0.1036 \mathrm{x}_{\mathrm{i}}\right)$ \\
\hline
\end{tabular}




\section{Summary:}

We set up reference case 1-D blanket, shield, and magnet models for fusion power plants. We used the ONEDANT neutronics code to calculate heating, tritium breeding ratio, neutron energy multiplication factor, inner magnet neutron fluence, and radiation dose for reference case zone thicknesses and variations from the reference case. We calculated linear and exponential fits to the data which can be used to calculate neutronics information for a wide range of first wall radii and blanket and shield thicknesses. This neutronics information, along with radiation limits, can be used in systems studies to help calculate appropriate sizes for $\mathrm{Li}-\mathrm{V}$, or cauldron blankets and shields for fusion energy power plants.

\section{References:}

1. S. W. Haney, Using and Programming the SuperCode, Lawrence Livermore National Laboratory, Livermore, $\mathrm{Ca}$ (unpublished work).

2. TWODANT-SYS One- and Two-Dimensional, Multigroup, DiscreteOrdinates Transport Code System, Radiation Shielding Information Center, Oak Ridge National Laboratory, CCC-547, Oak Ridge, Tennessee (1994).

3. L. El-Guebaly, University of Wisconsin, Private Communications (1995). 4. R. W. Werner, et. al., Synfuels from Fusion - Producing Hydrogen with the Tandem Mirror Reactor and Thermochemical Cycles, Lawrence Livermore National Laboratory Report UCID - 18909, Livermore, CA (1981).

\section{Acknowledgements:}

We wish to thank Laila El-Guebaly from the University of Wisconsin for help in getting started with the FRC blanket calculations. She suggested a starting composition and dimensions for the blanket and gave us radiation and heating limits. She later provided us with an example input file for the ONEDANT neutronics code and answered several questions we had regarding its use. We also wish to thank Hosny Ahaya of Argonne National Laboratory for his help in interpreting nuclear data files. This work was performed under the auspices of the U.S. Department of Energy by Lawrence Livermore National Laboratory under contract No. W-7405-ENG-48. 
Appendix 1

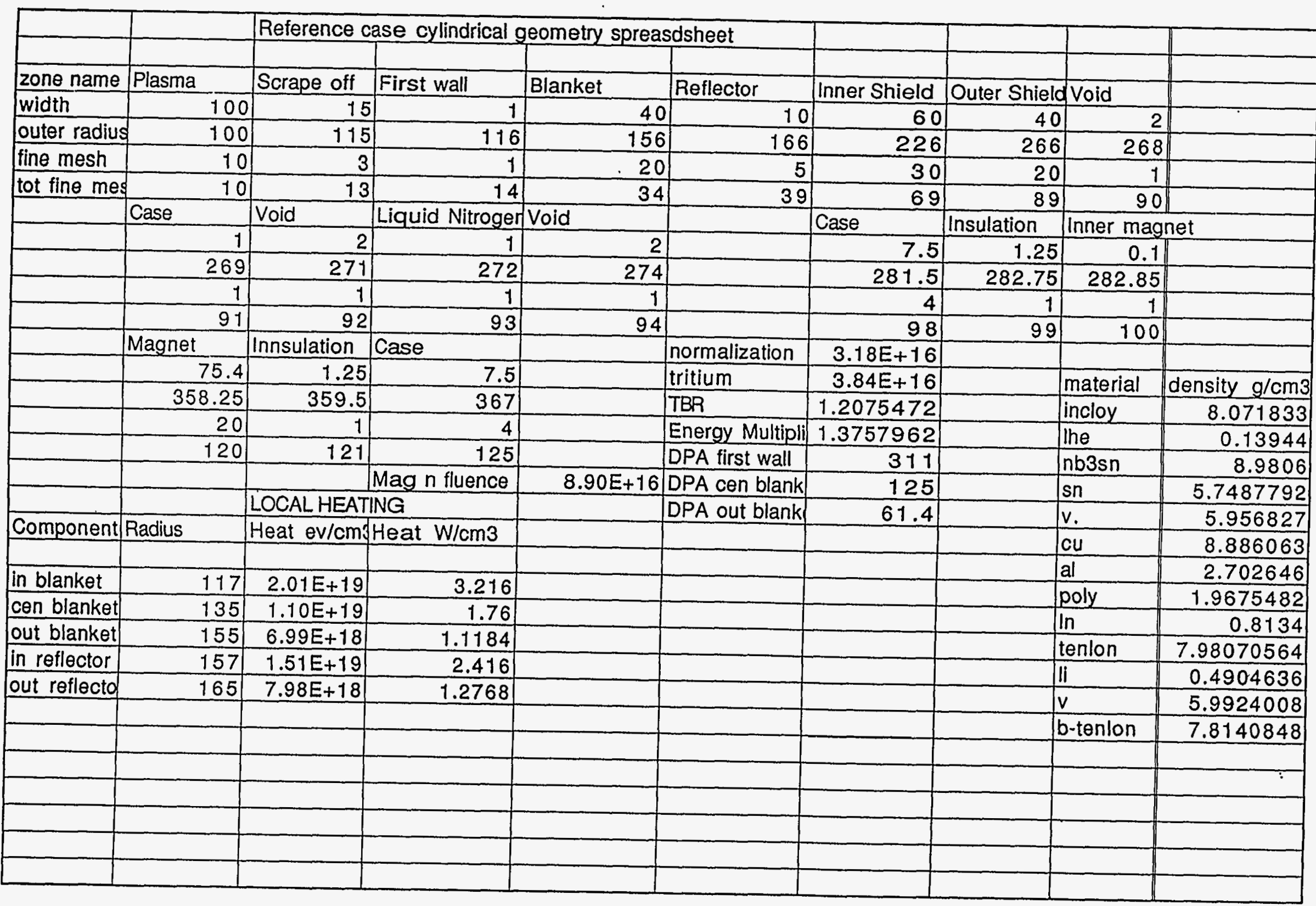




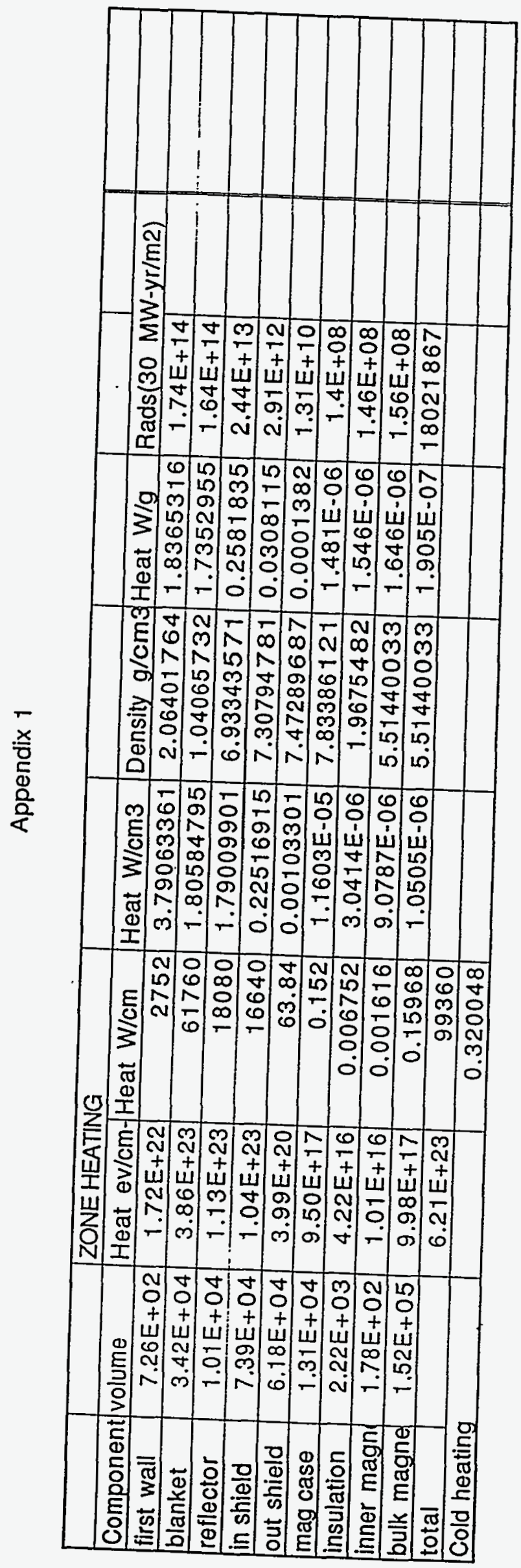

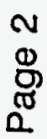

